\title{
Augmentation of transgenic expression by ultrasound-mediated liposome microbubble destruction
}

\author{
ZHI-YI CHEN $^{1 *}$, XIAO-FANG SUN ${ }^{2}$, JIAN-QIAO LIU ${ }^{2 *}$, BING SI-TU $^{3}$, RI-XIANG QIU ${ }^{3}$, KUN LIANG $^{2}$ \\ JIAN-HUA LIU ${ }^{4}$, WEI-XIANG LIANG ${ }^{1}$, XIN-XIN ZHOU $^{1}$, HUA ZHANG $^{1}$ and JIANG-XIU YU ${ }^{1}$ \\ ${ }^{1}$ Department of Medical Ultrasound, The Third Affiliated Hospital of Guangzhou Medical University, Guangzhou 510150; \\ ${ }^{2}$ Department of Obstetrics and Gynecology, Guangzhou Research Institute of Obstetrics and Gynecology, \\ The Third Affiliated Hospital of Guangzhou Medical University, Guangzhou 510150; ${ }^{3}$ Department of Pharmacy, \\ The Third Affiliated Hospital of Guangzhou Medical University, Guangzhou 510150; \\ ${ }^{4}$ Department of Function Imaging and Ultrasonography, Guangzhou First Municipal People's Hospital, \\ Guangzhou Medical College, Guangzhou 510180, P.R. China
}

Received November 8, 2011; Accepted January 11, 2012

DOI: $10.3892 / \mathrm{mmr} .2012 .766$

\begin{abstract}
Non-invasive, efficient and tissue-specific transgenic technologies could be valuable in gene therapy. Although non-viral carriers may be safer and cheaper, they have a much lower transfection efficiency than viral gene carriers. The present study was designed to test the transgenic expression and safety of red fluorescent protein (RFP) in HeLa cells in vitro and in transplanted tumors of nude mice in vivo under ultrasound-mediated liposome microbubble destruction (UMLMD) conditions. Plasmids containing RFP were gently mixed with liposome microbubbles (LMs). The mixture was added to HeLa cells or injected into BALB/c mice by the tail vein under various ultrasound exposure and LM parameters, and then the transfection efficiencies were examined. The results in vivo and in vitro demonstrated that, following a comparison of the plasmid group, the ultrasound + plasmid group and the LM + plasmid group, UMLMD significantly increased the transgenic expression $(\mathrm{P}<0.01)$ without causing any apparent detrimental effect. From the study, we concluded that UMLMD could be a non-invasive, effective and promising non-viral technique for gene therapy and transgenic research.
\end{abstract}

\section{Introduction}

Gene therapy has a broad application prospect in the treatment of cancer and genetic diseases, but its application is limited by

Correspondence to: Dr Zhi-Yi Chen, Department of Medical Ultrasound, The Third Affiliated Hospital of Guangzhou Medical University, 63 DuoBao Lu, Guangzhou 510150, P.R. China

E-mail: winchen@vip.126.com

*Contributed equally

Key words: ultrasound, sonoporation, microbubble, non-viral transfection the potential danger of virus vectors or the low transfection efficiency of non-virus vectors $(1,2)$. To enhance the transgenic expression of the non-viral gene transfer system, the transgenic technology of ultrasound-mediated gene transfection may be applied (3-6). Microbubbles, which act as contrast agents for medical ultrasound imaging, could improve the transfection efficiency (7-11). Furthermore, microbubbles excited by ultrasound exposure may temporarily 'open' the cell membranes of nearby cells allowing plasmid DNA to be delivered into cells. Subsequently, the cells could reseal themselves and maintain their vitality (12).

While liposomes have a very low immunogenicity, liposome microbubbles (LMs) are a research hotspot that has developed rapidly in recent years, which is capable of targeting increasing specific drug treatment or gene transfection (7,13-17). However, a number of factors could affect the transfection efficiency, including the ultrasonic intensity, the duration of ultrasound treatment and the microbubble concentration. Although the application of sonoporation has been optimized in certain studies, the delivery of drugs is often difficult to control and has been observed in a number of cases to have numerous unwanted side effects. Proteins with high wavelength emission spectra, including red fluorescent protein (RFP), may be a better choice as a reporter gene (18). However, few cases exist in the literature regarding in vitro and in vivo optimization using an RFP reporter gene. The effects of LM on transgenic expression have not been systematically assessed, and the applications of ultrasound-mediated liposome microbubble destruction (UMLMD) have not yet been optimized. However, we suggest this technique effectively transfers genes. In previous experiments $(19,20)$, a significant problem arose from the application of the non-viral gene transfer system of UMLMD. However, this UMLMD technique has not been studied thus far as a delivery system.

For this reason, we established a method that is costeffective and safer than viral carrier gene therapy. The present study utilized the method of UMLMD to examine the transfection efficiency and safety of RFP in vivo and in vitro, while 
assessing the safety and efficacy of UMLMD. Our purpose was to obtain optimal transgenic expression with the lowest cell or tissue injury, in order to lay the foundation for efficient, targeted and non-invasive gene therapy.

\section{Materials and methods}

Cell culture. HeLa cells were obtained from the China Center for Type Culture Collection (CCTCC) and incubated in Dulbecco's modified Eagle's medium (DMEM, Gibco, Grand Island, NY, USA) with $10 \%$ fetal bovine serum (FBS, Gibco), $100 \mathrm{U} / \mathrm{ml}$ penicillin and $100 \mu \mathrm{g} / \mathrm{ml}$ streptomycin at $37^{\circ} \mathrm{C}$ in a humidified environment of $5 \% \mathrm{CO}_{2} / 95 \%$ air. The total cell count was determined using a hemocytometer (Burker Turk, The Netherlands). The initial cell viability was determined by exclusion with trypan blue dye (Sigma-Aldrich, St. Louis, MO, USA). Exponentially growing cells were used in all experiments.

Plasmid DNA. The expression vector for the RFP gene plasmid (pDsRed-Express, Clontech, BD Biosciences, Billerica, MA, USA) had an excitation and emission maxima occurring at $557 \mathrm{~nm}$ and $579 \mathrm{~nm}$, respectively. The plasmid DNA was obtained from DH5 $\alpha$ Escherichia coli (Invitrogen, Carlsbad, CA, USA) and was prepared using a Qiaquick kit (Qiagen, Crawley, UK) according to the manufacturer's instructions. The absorbance ratio at the wavelength of 260-280 $\mathrm{nm}$ for the plasmid DNA solution was measured to be between 1.8 and 2.0.

Preparation of LMs. LMs were kindly provided by the Department of Ultrasonography of Xinqiao Hospital, The Third Military Medical University, Chongqing, China. The LMs were prepared using mechanical agitation. The activation device was refitted from an amalgamator (ST-D, Beijing AT\&M Biomaterials Co., Ltd, Beijing, China) at a calibration rate of $(4,500 \pm 100)$ oscillations/min for $45 \mathrm{sec}$, using a reciprocating motion and an oscillation amplitude of $(15 \pm 1) \mathrm{mm}$.

\section{In vitro studies}

Experimental apparatus. In vitro experiments were performed in an exposure water tank. The tank contained deionized water with an ultrasound transducer (Accusonic, Metron Medical Australia Pty., Ltd., Carrum Downs, Australia) fixed at the bottom. The culture plate was placed on the center of the transducer. The culture plate was covered with the lid to prevent it from being polluted by the surrounding environment. The experiments were performed on a sterile decontamination bench. To prevent the nearby wells from being affected by ultrasound irradiation, cells were planted in only six of 24-well culture plates. During ultrasound irradiation, the plate was agitated slowly, while remaining close to the transducer surface.

Experimental grouping and processes. HeLa cells were conventionally harvested by trypsinization and resuspended in a concentration of $5.0 \times 10^{6}$ cells $/ \mathrm{ml}$ in DMEM (300 $\mu 1$, non-FBS). Firstly, cells were irradiated using various ultrasound parameters and microbubble concentrations. Prior to ultrasound irradiation, LMs were mixed with $0.9 \%$ saline at various concentrations (v/v; 3, 6 and 10\%). LM suspension (150 $\mu \mathrm{l})$ and plasmid solution (final concentration $1.0 \mu \mathrm{g} / \mu \mathrm{l}$ ) were fully mixed to prepare the LM/plasmid complexes.
Ultrasound parameters were set as: ultrasonic frequency of $1 \mathrm{MHz}$, pulse repetition frequency of $100 \mathrm{~Hz}, 20 \%$ duty cycle, and ultrasound intensities of $0.8,1.0$ and $1.2 \mathrm{~W} / \mathrm{cm}^{2}$ with 3-min exposure.

To investigate the effect of LMs on the transfection and ultrasound exposure time, experiments were divided into the following groups: plasmid DNA group $(n=3)$; plasmid + ultrasound group, 3 wells irradiated for $1 \mathrm{~min}$ and 3 wells irradiated for $3 \mathrm{~min}$; LM + plasmid group $(\mathrm{n}=3)$; plasmid + LM + ultrasound group (P+ UMLMD), 3 wells irradiated for $1 \mathrm{~min}$ and 3 wells irradiated for $3 \mathrm{~min}$. Plasmid solution (150 $\mu \mathrm{l})(10 \mu \mathrm{g} /$ well) or LM suspension $(6 \%)$ or LM/plasmid complexes (as described above, LM concentration of $6 \%$ ) were added to the culture plate. The ultrasound intensity was fixed at $1.0 \mathrm{~W} / \mathrm{cm}^{2}$. When all the processes were completed, the culture plates were removed from the tank, wiped dry and returned to the incubator. A total of $8 \mathrm{~h}$ following ultrasound irradiation, the medium was replaced by one containing $10 \%$ FBS for continuous cultivation.

Cell viability and transgenic expression. Forty-eight hours after ultrasound irradiation, the RFP expression was observed using a fluorescence contrast phase microscope (IX71, Olympus, Japan). The cells were then harvested to assess the cell viability and the transgenic expression using different samples of the same cell suspension.

Propidium iodide (20 $\mu \mathrm{l})$ (PI, $40 \mu \mathrm{g} / \mathrm{ml}$, Sigma-Aldrich) was added into the cell suspension. Cells with PI were considered to be dead and cells without PI were considered to be alive. Following a $15 \mathrm{~min}$ incubation at room temperature, the cells were stored at $4^{\circ} \mathrm{C}$. Using flow cytometry (FACSCalibur, Becton Dickinson, Franklin Lakes, NJ, USA), the ratios of cells stained with or without PI were determined.

Approximately $1 \times 10^{5}$ cells were obtained from each sample for transgenic expression analysis, using a $550 \mathrm{~nm}$ wavelength excitation light and a $585 \pm 42 \mathrm{~nm}$ wavelength emission light to detect the red fluorescence. The transgenic expression was assessed as the number of cells expressing RFP per total number of survival cells. Data were analyzed using WinMDI software (version 2.8).

\section{In vivo studies}

Animal protocol. Animal handling and experimental procedures were approved by the Medical College Animal Experiments Committee. Four-to-six weeks old female Balb/c nu/nu mice, weighing 17-22 g, were maintained in specified pathogen-free (SPF) conditions throughout the experimental period.

Prior to animal modeling, HeLa cells were harvested, collected and centrifuged, and then resuspended in $100 \mu \mathrm{l}$ DMEM to prepare a single cell suspension. The mice were fixed on a superclean bench according to the principle of aseptic surgery, and inoculated subcutaneously into the flank with $2 \times 10^{6}$ cells per mouse following local sterilization. The mice were raised under the SPF condition following surgery, and were observed once every two days. Two weeks later, the experiments were initiated when the tumors reached a size of 5-10 mm.

Experimental grouping. A total of 20 mice were randomly divided into 4 experimental groups with 5 mice in each group: 
Table I. Transgenic expression of different ultrasound intensities and LM concentrations.

\begin{tabular}{lccc}
\hline $\begin{array}{l}\text { Ultrasound intensity } \\
\left(\mathrm{W} / \mathrm{cm}^{2}\right)\end{array}$ & \multicolumn{3}{c}{ LM concentration $(\%)$} \\
\cline { 2 - 4 } & \multicolumn{1}{c}{3} & 6 & 10 \\
\hline 0.8 & $9.03 \pm 2.33$ & $18.33 \pm 2.59^{\mathrm{a}}$ & $16.59 \pm 5.13$ \\
1.0 & $12.73 \pm 2.81$ & $31.18 \pm 5.48^{\mathrm{b}, \mathrm{d}}$ & $19.16 \pm 6.26$ \\
1.2 & $13.48 \pm 1.13$ & $24.47 \pm 3.24^{\mathrm{b}}$ & $13.32 \pm 1.03^{\mathrm{b}, \mathrm{c}}$
\end{tabular}

The data were presented as the mean values $\pm \mathrm{SEM}, \mathrm{n}=3$. With the fixed ultrasound intensity condition, ${ }^{\mathrm{a}} \mathrm{P}<0.05$, ${ }^{\mathrm{b}} \mathrm{P}<0.01$, as compared with the LM concentration of $3 \% ;{ }^{c} \mathrm{P}<0.01$, as compared with the LM concentration of $6 \%$. With the fixed LM concentration, ${ }^{\mathrm{d}} \mathrm{P}<0.05$, as compared with the ultrasound intensity of $0.8 \mathrm{~W} / \mathrm{cm}^{2}$. LM, liposome microbubble.

Plasmid group, injection of plasmid DNA $(50 \mu \mathrm{g} / 200 \mu \mathrm{l})$ alone; plasmid + ultrasound exposure group; LM + plasmid group, complexes of LM $(30 \mu \mathrm{l}, 6 \%)$ and plasmid $(50 \mu \mathrm{g})$ were gently agitated with PBS to a final volume of $200 \mu \mathrm{l}$ and directly injected; plasmid + LM + ultrasound group (P + UMLMD), complexes of plasmid DNA/LM were injected and followed by local ultrasound irradiation.

The plasmid DNA or LM/plasmid complexes were administered into the tail vein of the mice. The mice were anesthetized by diethylether and fixed on the flats. The tumors were subsequently sonicated using a transducer placed on the skin with a contact gel (Aquasonic 100, Parker Laboratories Inc., WI, USA). Ultrasound parameters were set at $3 \mathrm{MHz}$, $2 \mathrm{~W} / \mathrm{cm}^{2}, 2 \mathrm{~min}$ and $20 \%$ duty cycle. During the exposure, the ultrasound transducer was moved in a circular motion to ensure the whole tumor was exposed.

Analysis of transgenic expression in vivo. Three days following ultrasound treatment, the mice were sacrificed by cervical dislocation. The tumor specimens, surrounding tissues, the skin around the tumors, the hearts, livers and muscles, were immediately removed, embedded in optimal cutting temperature compound (OCT, Tissue-Tek, Sakura Finetek, Torrance, CA, USA), and stored at $-80^{\circ} \mathrm{C}$ until further analyses. Cross sections (10 $\mu \mathrm{m})$ were cut with a cryostat (CM1900, Leica, Germany) and affixed to glass slides. Fluorescence expression and distribution patterns were observed with confocal laser microscopy (Fluoview FV500, Olympus, Japan). The quantitative detection of transfection was performed using flow cytometry.

Histology. The specimens were fixed using formaldehyde, dehydrated with a graded alcohol series, and embedded in paraffin. Hematoxylin and eosin (H\&E) staining was performed on the specimens for histopathological evaluation of hemorrhage, necrosis and inflammation.

Statistical analysis. Statistical analyses were performed by the SPSS 13.0 software package (SPSS, Inc., Chicago, IL, USA). All values were expressed as the mean \pm SD. Analysis of variance with paired t-test and factorial design analysis of variance (ANOVA) test were used to determine the significance of the difference in a multiple comparison. If the ANOVA was significant, the Tukey's procedure was used as a post hoc test. $\mathrm{P}<0.05$ was considered to indicate a statistically significant difference.

\section{Results}

Effects of UMLMD on the transgenic expression in vitro. As shown in Table I, when the LM concentration was 3 and $10 \%$, there was no significant difference in the transgenic expression among the various ultrasound intensities $(\mathrm{F}=3.483, \mathrm{P}=0.099$; $\mathrm{F}=1.159, \mathrm{P}=0.375)$. When the $\mathrm{LM}$ concentration was $6 \%$, RFP expression of $1.0 \mathrm{~W} / \mathrm{cm}^{2}$ was higher than that of $0.8 \mathrm{~W} / \mathrm{cm}^{2}$ $(\mathrm{P}=0.017)$; however, the difference was not significant as compared with $1.2 \mathrm{~W} / \mathrm{cm}^{2}(\mathrm{P}=0.176)$. When the ultrasound intensity was fixed, the differences of transgenic expression among the various LM concentrations were significant $(\mathrm{F}=5.715, \mathrm{P}=0.041 ; \mathrm{F}=10.238, \mathrm{P}=0.012 ; \mathrm{F}=28.631, \mathrm{P}=0.001)$.

Cell injury of different ultrasound intensities and LM concentrations. Ultrasound exposure alone did not damage cells and the cell injury rate was $<14 \%$. When the ultrasound intensity was 0.8 or $1.0 \mathrm{~W} / \mathrm{cm}^{2}$, the differences among the various LM concentrations were not significant $(\mathrm{F}=1.072, \mathrm{P}=0.414$; $\mathrm{F}=0.376, \mathrm{P}=0.773)$. When the $\mathrm{LM}$ concentration was 3 or $6 \%$, there was no apparent cell damage in any experimental group. In addition, the differences between the ultrasound intensities were not significant $(\mathrm{F}=1.368, \mathrm{P}=0.324 ; \mathrm{F}=2.063, \mathrm{P}=0.208)$. The injury ratio was the highest with an ultrasound intensity of $1.2 \mathrm{~W} / \mathrm{cm}^{2}$ and $10 \% \mathrm{LM}(\mathrm{F}=7.070, \mathrm{P}=0.012 ; \mathrm{F}=6.612, \mathrm{P}=0.030$; Table II).

The factorial design analysis of variance indicated that the main effects analysis of 2 types of parameters were statistically significant (both $\mathrm{P}<0.01$ ), but there was no existing interaction between them $(\mathrm{P}>0.05)$. RFP expression was significantly higher in cells treated with an ultrasound intensity of $1.0 \mathrm{~W} / \mathrm{cm}^{2}$ and $6 \%$ LM, without causing any apparently adverse effect. According to these results, the subsequent experiments were performed under the optimal UMLMD conditions.

Augmentation of transgenic expression by UMLMD in vitro. Table III shows that without LM, the RFP expression of ultrasound irradiation alone was not significantly higher than that of the plasmid group alone ( $\mathrm{P}=0.816)$. The transgenic expression exposure of 3 min was not significantly higher than that of 1 min (paired t-test, $t=-2.443, \mathrm{P}=0.135$; Fig. $1 \mathrm{~A}$ and $\mathrm{B}$ ). LMs alone were insufficient to transfect the gene into cells without ultrasound irradiation. The differences had no statistical significance as compared with the plasmid alone or the ultrasound irradiation and plasmid group $(\mathrm{P}=0.095$ and $\mathrm{P}=0.312)$. 
Table II. Cell injury of different ultrasound intensities and LM concentrations.

\begin{tabular}{lcccc}
\hline $\begin{array}{l}\text { Ultrasound intensity } \\
\left(\mathrm{W} / \mathrm{cm}^{2}\right)\end{array}$ & \multicolumn{5}{c}{ LM concentration $(\%)$} \\
\cline { 2 - 5 } & - & 3 & 6 & 10 \\
\hline 0.8 & $10.57 \pm 1.37$ & $12.06 \pm 1.99$ & $12.82 \pm 1.95$ & $12.64 \pm 1.44$ \\
1.0 & $12.59 \pm 1.84$ & $13.50 \pm 1.93$ & $13.99 \pm 1.23$ & $14.71 \pm 4.08$ \\
1.2 & $13.95 \pm 1.86$ & $14.60 \pm 1.72$ & $15.85 \pm 2.21$ & $20.31 \pm 1.63^{\mathrm{a}, \mathrm{b}, \mathrm{c}}$
\end{tabular}

The data were presented as the mean values \pm SEM, $n=3$. With the fixed ultrasound intensity condition, ${ }^{a} \mathrm{P}<0.05$, as compared with the $\mathrm{LM}$ concentration of $6 \%$; ${ }^{b} \mathrm{P}<0.05$, as compared with $\mathrm{LM}(-)$. With the fixed $\mathrm{LM}$ concentration, ${ }^{\mathrm{C}} \mathrm{P}<0.05$, as compared with the ultrasound intensity of $0.8 \mathrm{~W} / \mathrm{cm}^{2}$. LM, liposome microbubble.

Table III. Effects of LM and ultrasound irradiation time on UMLMD.

\begin{tabular}{lcc}
\hline \multirow{2}{*}{$\begin{array}{l}\text { Ultrasound irradiation } \\
\text { time (min) }\end{array}$} & \multicolumn{2}{c}{ LM (\%) } \\
\cline { 2 - 3 } & & + \\
\hline- & $1.9 \pm 0.76$ & $4.48 \pm 1.18$ \\
1 & $2.73 \pm 1.39$ & $14.48 \pm 1.18^{\mathrm{a}, \mathrm{c}}$ \\
3 & $3.83 \pm 0.98$ & $31.18 \pm 5.48^{\mathrm{a}, \mathrm{b}, \mathrm{c}}$ \\
\hline
\end{tabular}

Data were presented as the mean values \pm SEM, $n=3$. UMLMD parameters: LM concentration of $6 \%$, ultrasound intensity of $1.0 \mathrm{~W} / \mathrm{cm}^{2}$. With the fixed LM condition, ${ }^{\text {a }}<<0.01$, as compared with ultrasound irradiation (-); ${ }^{\mathrm{b}} \mathrm{P}<0.01$, as compared with ultrasound irradiation time of $1 \mathrm{~min}$. With the fixed ultrasound irradiation condition, ${ }^{\mathrm{C}} \mathrm{P}<0.01$, as compared with LM (-). LM, liposome microbubble; UMLMD, ultrasound-mediated liposome microbubble destruction.

However, the cells that received an injection of plasmid with LM followed by ultrasound irradiation (P + UMLMD) demonstrated a significantly higher transgenic expression than any other group (all $\mathrm{P}<0.01$, Fig. 1C). Moreover, the transgenic expression for an exposure time of 3 min was significantly higher than that of $1 \mathrm{~min}$ (paired t-test, $\mathrm{t}=-5.714$, $\mathrm{P}=0.029$; Fig. 1D). These results demonstrated that LM had a markedly enhanced effect on the transgenic expression of UMLMD.

Targeted delivery and enhancement of transgenic expression by UMLMD in vivo. Without ultrasound irradiation, limited RFP expression in the plasmid injection alone group was detected $(1.83 \pm 1.21 \%)$, and the fluorescence signal was weak (Fig. 2A). The plasmid and LM injection group also demonstrated a similar fluorescence expression rate $(2.33 \pm 1.39 \%$, $\mathrm{P}=0.753$; Fig. 2B). The RFP expression rate of the plasmid injection and ultrasound irradiation group was $3.48 \pm 0.18 \%$; however, when compared with the plasmid injection alone, the difference was not significant $(\mathrm{P}=0.517)$. In addition, the majority of fluorescent protein expression detected was distributed in the superficial area of the tumors (Fig. 2C). However, when the plasmid was injected with LM followed by ultrasound irradiation, RFP expression increased significantly $(23.96 \pm 2.13 \%)$, with a stronger signal and a greater density (Fig. 2D). As compared with the other three groups, the differences were significant $(\mathrm{F}=172.954, \mathrm{P}<0.001$, all $\mathrm{P}<0.01)$.

Tissue specificity by UMLMD in vivo. None of the nude mice died during the experiment. Regardless of the ultrasound exposure, a weak RFP expression was evident in certain livers (Fig. 3A), hearts (Fig. 3B) and muscles (Fig. 3C), whereas other organ tissues had no significant expression.

Histological observation. H\&E staining revealed that tissue damage was not observed in the tumors (Fig. 4A) and other organs (Fig. 4B-D), which remained intact. Moreover, the results demonstrated no abnormalities, including inflammation or degeneration, in any tissues.

\section{Discussion}

For gene therapy, although the viral vector is an effective transgenic method for gene transfection, its safety and side-effects remain a concern for clinical application. In non-viral transgenic technologies, UMLMD is a simple, non-invasive method. It can focus on specific tissues or organs directly and it is beneficial for the localization of the gene of interest. It is safer than other methods, and may be used in clinical applications in the near future. Cell permeability may be transiently changed by sonoporation, in order that macromolecules, including the plasmid DNA are able to enter the cells instantaneously $(8,9,21,22)$. Results of previous studies showed that $(8-11,23)$ the addition of microbubbles were capable of promoting transgenic expression. However, if sonoporation was extremely strong, it would lead to apparent cell death. The ideal concentration of microbubbles should deliver the drug or gene into cells with the greatest efficiency without lethal effect.

A significant problem from the application of the non-viral gene transfer system of UMLMD occurred in our study. This technology provided a new promising approach for gene delivery in vitro and in vivo. However, this technique for the delivery system of shRNA in vitro has yet to be optimized. In the present study, parameters for the delivery system were optimized. Fig. 1 shows that transfection efficiency was affected by the transfection parameters, including ultrasound intensity and exposure time. The optimal conditions were capable of achieving the highest transfection efficiency. Our results demonstrated that 



Figure 1. Effects of LM and exposure time on the transgenic expression of red fluorescent protein in vitro; as mentioned in Materials and methods, in vivo experimental grouping. (A) Plasmid injection and ultrasound exposure $1 \mathrm{~min}$; (B) plasmid injection and ultrasound exposure 3 min. As compared with Fig. 1A, the transgenic expression of red fluorescent protein in vitro was not significantly higher than that of $1 \mathrm{~min}(\mathrm{P}>0.05)$; (C) LM and plasmid injection followed by ultrasound exposure of $1 \mathrm{~min}, \mathrm{LM}$ alone was insufficient to transfect the genes into cells without ultrasound irradiation. (D) LM and plasmid injection followed by ultrasound exposure of $3 \mathrm{~min}$. As compared with Fig. 1C, the transgenic expression of red fluorescent protein in vitro was significantly higher than that of $1 \mathrm{~min}(\mathrm{P}<0.01)$. Bar, $100 \mu \mathrm{m}$. LM, liposome microbubble.
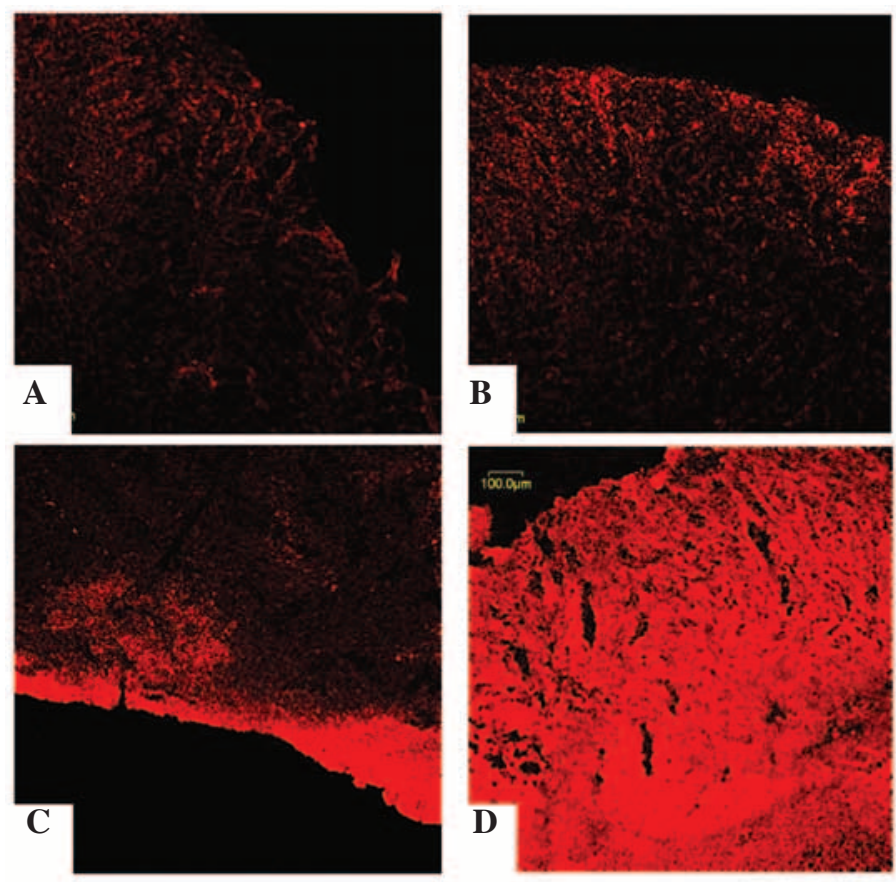

Figure 2. Expression of red fluorescent protein in the transplantation tumors of nude mice; as mentioned in Materials and methods, in vivo experimental grouping. (A) Plasmid injection alone; (B) LM and plasmid injection; (C) plasmid injection and ultrasound exposure. As compared with Fig. 2A and B, the transgenic expression of red fluorescent protein was not significant. (D) LM/plasmid complexes injection followed by ultrasound exposure (P + UMLMD). Expression of red fluorescent protein increased significantly with a strong signal and a greater density in the P + UMLMD group as compared with the other groups. Bar, $100 \mu \mathrm{m}$. LM, liposome microbubble; UMLMD, ultrasound-mediated liposome microbubble destruction.

the RFP transgenic expression was the highest when LMs were combined with ultrasound exposure, while the cells were not markedly injured. Moreover, the UMLMD was also an effective and tumor-specific transgenic technology in vivo. Plasmid DNA was rapidly transferred to transplanted tumors even under the conditions in the bloodstream and serum, and transgenic expression depended on the ultrasound irradiation site. In this study, the reporter gene was RFP, whose provocation and emission wavelength were longer than other genes such as GFP, and its emission peak was located beyond the scope of the fluorescence background generated by the medium and tissue culture equipment. RFP, which is expressed in mammalian cells effectively, has a high signal-to-noise ratio, is not easy to induce fluorescence quenching, and has a high conversion efficiency. The 

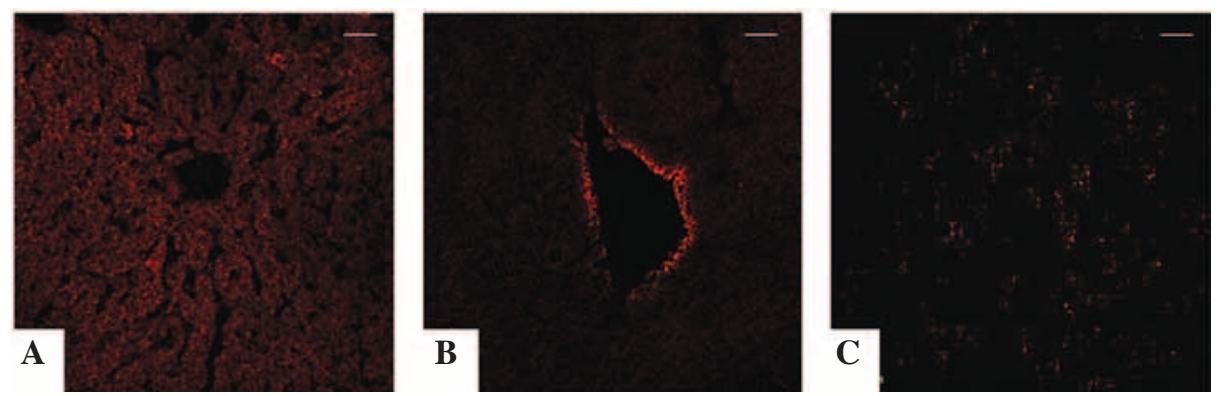

Figure 3. Confocal laser scanning microscopic images of the non-targeted organs by UMLMD treatment; as mentioned in Materials and methods, the in vivo experimental grouping. (A) liver, (B) heart, (C) muscle. In the P + UMLMD group, there was weak red fluorescence expression in certain livers, hearts and muscles, while other organ tissues had no significant expression of red fluorescence. Bar, $100 \mu \mathrm{m}$. UMLMD, ultrasound-mediated liposome microbubble destruction.
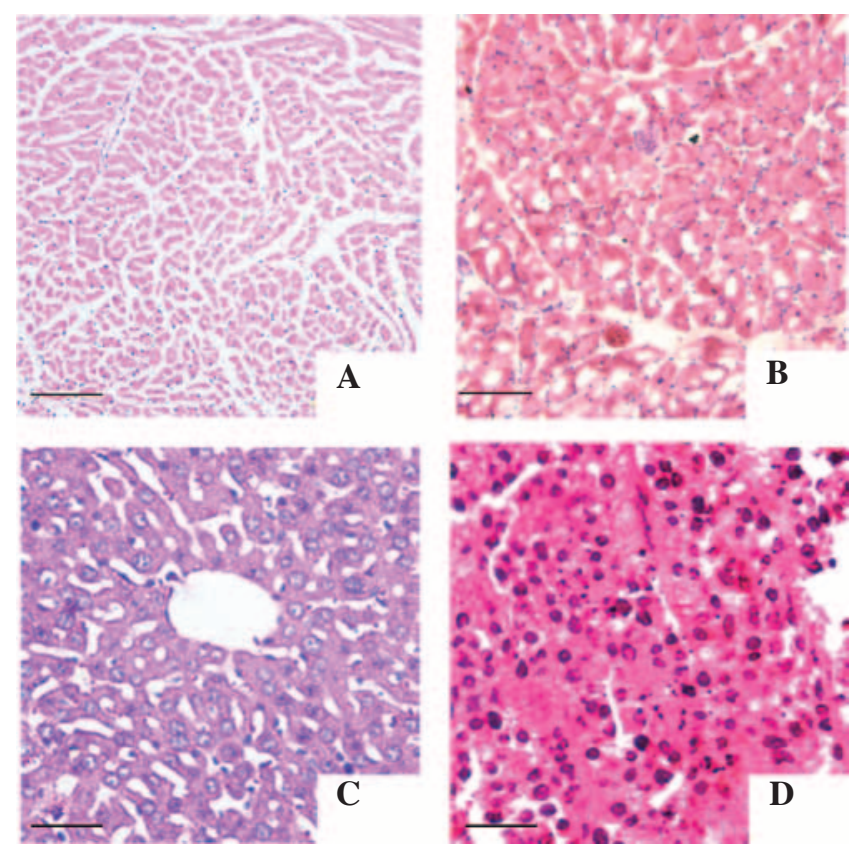

Figure 4. Histological observations; as mentioned in Materials and methods in the in vivo experimental grouping. (A) Heart, bar, $100 \mu \mathrm{m}$, (B) Muscle, bar, $100 \mu \mathrm{m}$, (C) Liver, bar, $50 \mu \mathrm{m}$, (D) Tumor, bar, $100 \mu \mathrm{m}$; H\&E staining demonstrated that tissue damage, inflammation and degeneration were not observed in the representative sections.

far-red emissions may be preferred for certain applications due to the lower background autofluorescence in certain tissues (18).

Microbubbles are not only useful as contrast agents, but are also widely used in therapeutic applications. Microbubbles may amplify the cavitation effect of ultrasound, particularly in targeting drug and gene delivery in vitro and in vivo. However, the mean diameter of conventional microbubbles is approximately $2.0-4.5 \mu \mathrm{m}$. Tsunoda et al (24) reported that several mice died immediately following i.v. injection due to lethal embolisms in certain organs. It is therefore necessary to develop novel microbubbles and new techniques to improve the transfection efficiency. LMs have been used in clinical ultrasound diagnosis and treatment safely and stably, with low immunogenicity and cytotoxicity. However, LM alone was insufficient to transfect the gene into cells without ultrasound exposure. The transfection efficiency was enhanced by the novel combination of LM and ultrasound exposure via the mechanism of sonoporation. In this study, LMs were composed of polyethyleneglicol (PEG) and liposome, which independently have been used in clinical ultrasound diagnosis and treatment safely and stably with a long intravascular half-life (25). The mean diameter of LMs was smaller than that of red blood cells (26), meaning that it was capable of reaching peripheral tissues and passing through capillary vessels. Therefore, the LMs were used repeatedly to further enhance and maintain the gene expression. Moreover, under ultrasound irradiation, LM significantly promoted transgenic expression. However, when excessive LM was added, the transgenic expression declined and caused cell damage (27). Similarly to another study (28), various ultrasound intensities and irradiation times had a great impact on UMLMD. As the matching of specific ultrasound parameters and transgenic technologies were different, the optimal conditions of various cell types were also different. The ultrasound response differences among various cell lines also lead to different conclusions (29). Furthermore, LM could be developed as a 
gene transfection tool and delivered into specific tissues or organs. Significantly, the in vivo study concerning the targeted delivery of LMs and the effects of ultrasound allowed for further investigation into the transfection of transplantation tumors in nude mice in our laboratory. For this type of gene transfer system, modulating the biodistribution of the LMs and the plasmid DNA in the systemic injection is crucial. Unlike the in vitro study, ultrasound exposure of $3 \mathrm{MHz}$ facilitated the degree of tumor targeting, indicating the significance of localizing ultrasonic energy in the tumor volume (30). Ultrasound irradiation had no significant impact on other organs. Our results demonstrated that significant transgenic expression and noninvasive, targeting gene transfer were obtained by LM and plasmid DNA complex injections with ultrasound irradiation.

However, there were certain limitations to our study, and further studies are required to investigate the mode of injection and the time point and dose-response correlation in larger animal models. In addition, the optimal conditions in the in vivo experiment, including ultrasound parameters, concentration of LMs and plasmid DNA, are likely to be different from the in vitro experiment. Furthermore, to enhance the tissue specificity of the transgenic expression, several modifications are required and should be studied. For example, certain peptides or receptors on the surface of LMs may be investigated and may specify the gene expression in certain tissues.

In conclusion, in this study, UMLMD was found to enhance the transgenic efficiency without causing any apparent detrimental effect. If the appropriate target gene is added, this transgenic technology is expected to become an effective strategy for human gene therapy, and provide a non-invasive, safe and promising technique for transgenic research.

\section{Acknowledgements}

This study was supported by the Medical Research Foundation of Guangdong Province (No. A2010270), the Research Projects of Guangzhou Education Bureau (No. 10A242), the Youth Research Support Projects of the Third Affiliated Hospital of Guangzhou Medical University (No. 2011Y02) and the Medical Research Foundation of Liwan District (No. 20111213067).

\section{References}

1. Mehier-Humbert S and Guy RH: Physical methods for gene transfer: improving the kinetics of gene delivery into cells. Adv Drug Deliv Rev 57: 733-753, 2005.

2. Miller DL, Pislar SV and Greenleaf JE: Sonoporation: mechanical DNA delivery by ultrasonic cavitation. Somat Cell Mol Genet 27: 115-134, 2002.

3. Azuma H, Tomita N, Sakamoto T, et al: Marked regression of liver metastasis by combined therapy of ultrasound mediated $\mathrm{NF} \kappa \mathrm{B}$-decoy transfer and transportal injection of paclitaxel, in mouse. Int J Cancer 122: 1645-1656, 2008.

4. Sheyn D, Kimelman-Bleich N, Pelled G, Zilberman Y, Gazit D and Gazit Z: Ultrasound-based nonviral gene delivery induces bone formation in vivo. Gene Ther 15: 257-266, 2008.

5. Feril LB Jr, Ogawa R, Tachibana K and Kondo T: Optimized ultrasound-mediated gene transfection in cancer cells. Cancer Sci 97: 1111-1114, 2006

6. Watanabe A, Otake R, Nozaki T, et al: Effects of microbubbles on ultrasound-mediated gene transfer in human prostate cancer PC3 cells: comparison among Levovist, YM454, and MRX-815H. Cancer Lett 265: 107-112, 2008.
7. Li T, Tachibana K, Kuroki M and Kuroki M: Gene transfer with echo-enhanced contrast agents: comparison between Albunex, Optison, and Levovist in mice-initial results. Radiology 229: 423-428, 2003.

8. Taniyama Y, Tachibana K, Hiraoka K, et al: Development of safe and efficient novel nonviral gene transfer using ultrasound: enhancement of transfection efficiency of naked plasmid DNA in skeletal muscle. Gene Ther 9: 372-380, 2002.

9. Duvshani-Eshet M, Adam D and Machluf M: The effects of albumin-coated microbubbles in DNA delivery mediated by therapeutic ultrasound. J Control Release 112: 156-166, 2006

10. Manome Y, Nakayama N, Nakayama K and Furuhata H: Insonation facilitates plasmid DNA transfection into the central nervous system and microbubbles enhance the effect. Ultrasound Med Biol 31: 693-702, 2005.

11. Hernot $S$ and Klibanov AL: Microbubbles in ultrasound-triggered drug and gene delivery. Adv Drug Deliv Rev 60: 1153-1166, 2008.

12. Yang F, Gu N, Chen D, et al: Experimental study on cell self-sealing during sonoporation. J Control Release 131: 205-210, 2008.

13. Suzuki R, Takizawa T, Negishi Y, Utoguchi N and Maruyama K: Effective gene delivery with novel liposomal bubbles and ultrasonic destruction technology. Int J Pharm 354: 49-55, 2008.

14. Vandenbroucke RE, Lentacker I, Demeester J, De Smedt SC and Sanders NN: Ultrasound assisted siRNA delivery using PEG-siPlex loaded microbubbles. J Control Release 126: 265-273, 2008.

15. Leong-Poi H, Kuliszewski MA, Lekas M, et al: Therapeutic arteriogenesis by ultrasound-mediated VEGF165 plasmid gene delivery to chronically ischemic skeletal muscle. Circ Res 101: 295-303, 2007.

16. Unger EC, Porter T, Culp W, Labell R, Matsunaga T and Zutshi R. Therapeutic applications of lipid-coated microbubbles. Adv Drug Deliv Rev 56: 1291-1314, 2004.

17. Luo YK, Zhao YZ, Lu CT, Tang J and Li XK: Application of ultrasonic gas-filled liposomes in enhancing transfer for breast cancer-related antisense oligonucleotides: an experimental study. J Liposome Res 18: 341-351, 2008.

18. Yurchenko E, Friedman H, Hay V, Peterson A and Piccirillo CA: Ubiquitous expression of mRFP-1 in vivo by site-directed transgenesis. Transgenic Res 16: 29-40, 2007.

19. Chen ZY, Xie MX, Wang X and Lu Q: Effects of lipid shell microbubble on ultrasound mediated EGFP gene delivery to transplanted tumors: initial experience. Chin Ger J Clin Oncol 7: 424-428, 2008.

20. Chen ZY, Liang K, Liu JH, et al: Enhancement of survivin gene downregulation and cell apoptosis by a novel combination: liposome microbubble and ultrasound exposure. Med Oncol 26: 491-500, 2009.

21. Pitt WG, Husseini GA and Staples BJ: Ultrasonic drug delivery: a general review. Expert Opin Drug Deliv 1: 37-56, 2004.

22. Newman CM and Bettinger T: Gene therapy progress and prospects: ultrasound for gene transfer. Gene Ther 14: 465-475, 2007.

23. Yoon CS, Jung HS, Kim TK, et al: Comparison of the efficiency and toxicity of sonoporation with branched polyethylenimine-mediated gene transfection in various cultured cell lines. J Drug Target 16: 773-779, 2008.

24. Tsunoda S, Mazda O, Oda Y, et al: Sonoporation using microbubble BR14 promotes pDNA/siRNA transduction to murine heart. Biochem Biophys Res Commun 336: 118-127, 2005.

25. Bian AN, Gao YH,Tan KB, et al: Preparation of human hepatocellular carcinoma-targeted liposome microbubbles and their immunological properties. World J Gastroenterol 10: 3424-3427, 2004.

26. Liu P, Gao YH, Tan KB, Liu Z and Zuo S: Grey scale enhancement of rabbit liver and kidney by intravenous injection of a new lipid-coated ultrasound contrast agent. World J Gastroenterol 10: 2369-2372, 2004.

27. Rahim A, Taylor SL, Bush NL, ter Haar GR, Bamber JC and Porter CD: Physical parameters affecting ultrasound/microbubble-mediated gene delivery efficiency in vitro. Ultrasound Med Biol 32: 1269-1279, 2006.

28. Liang HD, Lu QL, Xue SA, ter Haar GR, Bamber JC and Porter CD. Optimisation of ultrasound-mediated gene transfer (sonoporation) in skeletal muscle cells. Ultrasound Med Biol 30: 1523-1529, 2004.

29. Larina IV, Evers BM and Esenaliev RO: Optimal drug and gene delivery in cancer cells by ultrasound-induced cavitation. Anticancer Res 25: 149-156, 2005.

30. Gao ZG, Fain HD and Rapoport N: Controlled and targeted tumor chemotherapy by micellar-encapsulated drug and ultrasound. J Control Release 102: 203-222, 2005. 